\title{
Iran's international collaborative articles in medical sciences
}

\author{
Habibolah Rezaei ${ }^{1,2}$, Alireza Yousefi ${ }^{3}$, Bagher Larijani ${ }^{4}$, Reza Dehnavieh ${ }^{5}$, Nima Rezaei ${ }^{6}$, \\ Maryam Kazemi ${ }^{7}$, Peyman Adibi*8
}

\section{Abstract}

Background: Forecasting is the process of predicting future behavior. In reviewing databases, no predicted value associated with international collaboration publications in Iran was found. Thus, the present study aimed at forecasting Iran's international collaborative articles in medical sciences.

Methods: The number of Iran's articles and international collaborative articles in medical sciences written over 56 years was extracted from SCOPUS. Data were extracted from 1960 up to 2016. The time series method was used for forecasting using the Minitab software Version 17.

Results: There was no increase in the number of medical articles from Iran from 1960 to 2001. However, the data showed incremental growth between 2001 and 2016. This was similar to Iran's medical sciences international collaboration articles. In 2016 , the percentage of Iran's international collaboration articles was 15.2, which is expected to reach 19.9 in 2025.

Conclusion: An investigation was performed on the number of international collaboration articles in the field of medical sciences in Iran. Future trends show an incremental growth. The number of Iran's articles can be increased with international cooperation. However, an increase or decrease in Iran's articles without international cooperation has to be investigated.

Keywords: Forecasting, International cooperation, Interrupted time series analysis, Medicine, Iran

Conflicts of Interest: None declared

Funding: Deputy of Education of the Ministry of Health and Medical Education and Vice Chancellor for Research of Isfahan University of Medical Sciences

\section{*This work has been published under CC BY-NC-SA 1.0 license.}

Copyright $\odot$ Iran University of Medical Sciences

Cite this article as: Rezaei H, Yousefi A, Larijani B, Dehnavieh R, Rezaei N, Kazemi M, Adibi P. Iran's international collaborative articles in medical sciences. Med J Islam Repub Iran. 2019 (17 Aug);33:84. https://doi.org/10.47176/mjiri.33.84

\section{Introduction}

Change is happening at an ever faster rate today. Thus, it is necessary for governments, businesses, organizations, and the public to improve their understanding of change and the future, and through this, they can positively influence the future (1). To assist people in choosing and creating their most desirable future, futurists discover possible, probable, and preferable futures (2). There are a number of methods that futurists use, such as forecasting with time series and trend analysis $(3,4)$. Forecasting is the process of predicting future behavior (4). In forecasting, the future is predicted from the past (5). Trend analysis is a method of future study (4). Trend analysis of research publication
Corresponding author:Dr Peyman Adibi, adibi@med.mui.ac.ir

1. Medical Education, Medical Education Research Center, Isfahan University of Medical Sciences, Isfahan, Iran

2. Cellular \& Molecular Research Center, Yasuj University of Medical Sciences, Yasuj, Iran

3. Medical Education Research Center, Isfahan University of Medical Sciences, Isfahan, Iran

4. Medical Ethics and History of Medicine Research Center, Tehran University of Medical Sciences, Tehran, Iran

5. Research Center for Modeling in Health, Institute for Futures Studies in Health, Kerman University of Medical Sciences, Kerman, Iran

6. Department of Immunology, School of Medicine, Molecular Immunology Research Center, Tehran University of Medical Sciences, Tehran, Iran

7. Department of Biostatistics and Epidemiology, School of Health, Isfahan University of Medical Sciences, Isfahan, Iran

8. Integrative Functional Gastroenterology Research Center, Isfahan University of Medical Sciences, Isfahan, Iran $\uparrow$ What is "already known" in this topic:

The annual number of Iran's articles and its number of international collaborative articles in medical sciences has already been known.

\section{$\rightarrow$ What this article adds:}

In this study, the trend of number of medical sciences articles from 1960 to 2016, the number of medical sciences articles, and the number of medical sciences international collaboration articles from 2017 to 2025 in Iran have been forecasted. 
activity within a period of time provides information to decision-makers and researchers. It provides a more informed understanding of development in a field and allows for simulation and scenario building using forecasting methods (4).

International cooperation has a long history. International cooperation is an important source of knowledge in outstanding science in Europe since the 1950s (6). International cooperation network was initially launched by several European countries and the United States. Currently, all countries are involved in international cooperation (7). International cooperation in science has grown in recent years (7-9). For example, studies show that the contribution of South Korea, China, and Japan in writing international collaborative articles has increased (7, 10, and 11). According to the SCIMago ranking system, the percentage of international collaborative articles in the United States, China, United Kingdom, Germany, Japan, and Iran was $17.59 \%, 17.59 \%, 50.57 \%, 47.79 \%, 26.22 \%$, and $21.02 \%$, respectively, in 2014 (12-17).

Forecasting international collaboration scientific publications is important, but it has not received enough attention. One study showed that India's number of overall scientific publications has increased significantly in recent years, with 11976 in 1995, 39407 in 2005, and 99771 in 2012. It is expected that India generate 178796 research papers by 2020 (4). In that study, it was mentioned that in India, the number of internationally collaborated scientific publications was 1357 in 1995, 11475 in 2005, and 30 213 in 2012 and predicted that the number of India's research papers increase to 62599 in the year 2020. The role of international collaboration in Indian scientific publications has shifted from a position of little importance to that of significant importance (4). Indian publication activity shows a significant annual growth. The growth of internationally collaborative papers is faster than the growth of overall Indian papers (4).

Information about the article presented above applies to all fields. The statistics of international collaboration publications in the field of medical sciences need special attention. In this regard, one study was conducted on 50 medical institutes of India and found that the growth of national research output has doubled and the growth of research with international collaboration has grown 4 times during 2003 and 2012 (18). In reviewing the data- bases, no predicted value associated with medical sciences international collaboration publications in Iran was found. Iran plans to improve the internationalization of medical sciences. One component of internationalization is international collaborative articles. If Iran wishes to expand its symbol and compete with regional countries, it should increase international collaboration. Iranian medical authorities would like to know where they will stand if the current trend continues in the articles and how much the articles should increase. Therefore, the present study aimed at forecasting Iran's international collaborative articles in medical sciences.

\section{Methods}

In this futures study, an explorative approach was used for forecasting. The trend analysis method was used for depicting the trends. A time series method was used for forecasting using Minitab software Version 17. Smoothing method and forecasts by Holt double exponential smoothing were used. This procedure can work well when a trend is present, but it can also serve as a general smoothing method (19). To minimize the sum of squared errors, we fitted an ARIMA $(0,2,2)$ model for Iran's number of medical sciences articles and its number of medical sciences international collaboration articles.

The number of articles and international collaborative articles in medical sciences written over 56 years was extracted from Scopus. Data were extracted from 1960 until 2016. The subject areas included dentistry, health profession, immunology and microbiology, medicine, neuroscience, nursing, pharmacology, toxicology and pharmaceutics, biochemistry, and genetics and molecular biology. The document types were articles, reviews, letters, notes, editorials, press articles, conference papers, and short surveys.

\section{Results}

Iran's number of articles and its number of international collaboration articles in the field of medical sciences were extracted from 1960 up to 2016. The number that Iran could reach by 2025 was also forecasted. Iran's number of medical sciences articles and its number of medical sciences international collaboration articles from 1960 to 2016 are displayed in Table 1.

As Table 1 demonstrates, in 2016, Iran's number of

\begin{tabular}{lcc}
\multicolumn{2}{l}{ Table 1. Iran's number of medical sciences articles and Iran's number of medical sciences international collaboration articles from 1960 to 2016} \\
\hline Year & Iran's number of medical sciences articles & Iran's number of medical Sciences international collaboration articles \\
\hline 2016 & 11621 & 1772 \\
2015 & 11485 & 1677 \\
2014 & 11768 & 1677 \\
2013 & 11716 & 1555 \\
2012 & 9891 & 1341 \\
2011 & 8172 & 1163 \\
2010 & 6189 & 939 \\
2009 & 5459 & 854 \\
2008 & 4515 & 752 \\
2007 & 3565 & 597 \\
2006 & 2707 & 421 \\
2005 & 1922 & 352 \\
2004 & 1115 & 257 \\
2003 & 919 & 207 \\
\hline
\end{tabular}




\begin{tabular}{|c|c|c|}
\hline Year & Iran's number of medical sciences articles & Iran's number of medical Sciences international collaboration articles \\
\hline 2001 & 355 & 60 \\
\hline 2000 & 309 & 64 \\
\hline 1999 & 269 & 47 \\
\hline 1998 & 235 & 56 \\
\hline 1997 & 241 & 40 \\
\hline 1996 & 170 & 40 \\
\hline 1995 & 122 & 18 \\
\hline 1994 & 92 & 14 \\
\hline 1993 & 87 & 20 \\
\hline 1992 & 66 & 14 \\
\hline 1991 & 54 & 6 \\
\hline 1990 & 56 & 9 \\
\hline 1989 & 67 & 5 \\
\hline 1988 & 44 & 2 \\
\hline 1987 & 41 & 6 \\
\hline 1986 & 40 & 6 \\
\hline 1985 & 44 & 5 \\
\hline 1984 & 37 & 10 \\
\hline 1983 & 53 & 9 \\
\hline 1982 & 55 & 10 \\
\hline 1981 & 82 & 13 \\
\hline 1980 & 111 & 14 \\
\hline 1979 & 186 & 22 \\
\hline 1978 & 250 & 24 \\
\hline 1977 & 240 & 24 \\
\hline 1976 & 200 & 20 \\
\hline 1975 & 175 & 20 \\
\hline 1974 & 179 & 9 \\
\hline 1973 & 168 & 16 \\
\hline 1972 & 58 & 15 \\
\hline 1971 & 45 & 12 \\
\hline 1970 & 40 & 6 \\
\hline 1969 & 28 & 6 \\
\hline 1968 & 22 & 1 \\
\hline 1967 & 31 & 5 \\
\hline 1966 & 12 & 2 \\
\hline 1965 & 8 & 2 \\
\hline 1964 & 7 & 3 \\
\hline 1963 & 2 & 0 \\
\hline 1962 & 12 & 4 \\
\hline 1961 & 7 & 2 \\
\hline 1960 & 4 & 1 \\
\hline
\end{tabular}

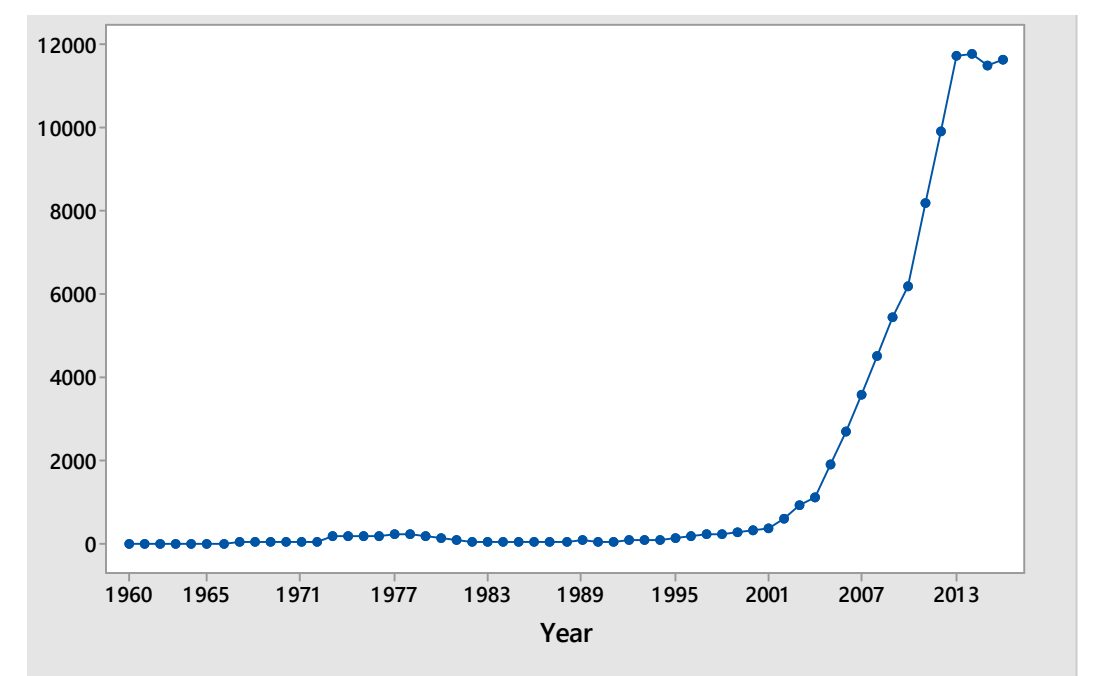

Diagram 1. Times series plot of Iran's number of medical sciences articles from 1960 to 2016

medical sciences articles was 11621 and its number of medical sciences international collaboration articles was 1172. Therefore, the percentage of international collabora- tion articles stood at 15.2.

The trend of Iran's number of medical sciences articles from 1960 to 2016 is displayed in Diagram 1. 
As Diagram 1 displays, Iran's number of medical sciences articles did not grow from 1960 to 2001. However, the trend shows an incremental growth from 2001 to 2016.

The trend of Iran's number of medical sciences international collaboration articles from 1960 to 2016 is shown in Diagram 2.

As Diagram 2 demonstrates, Iran's number of medical sciences international collaboration articles showed no growth from 1960 to 2001 . However, the trend shows an incremental growth from 2001 to 2016.

Then, forecasting was done using the available data. Forecasting for Iran's number of medical sciences articles and its number of medical sciences international collaboration articles from 2017 to 2025 are presented in Table 2 .

As Table 2 displays, in 2025, Iran's number of medical sciences articles will be 14580 and Iran's number of medical sciences international collaboration articles will be 2905. Therefore, the percentage of international collaboration is 19.9 .

Estimation of smoothing parameters and goodness of fit for models is demonstrated in Table 3.

As Table 3 displays, these models are appropriate. Also, after smoothing, the residuals diagram shows normality, variance stability, and independence of residuals for 2 models. Autocorrelation diagram also shows deleted autocorrelation in the residuals after exponential smoothing. Therefore, the models are appropriate.

\section{Discussion}

There has been increasing interest in research collaboration in recent years (20). Collaboration in science is already perceived as a national or regional phenomenon (21). Currently, collaboration has a wider scope between continents and often on a global scale (21). It is widely assumed that collaboration in research is 'a good thing' and it should be encouraged (22). Forecasting research publication activity provides information to decisionmakers (4). If Iran wants to expand its symbol and compete with regional countries, it should increase international collaboration. Therefore, the present study aimed at forecasting Iran's international collaborative articles in the field of medical sciences.

The results of the study showed that Iran's number of medical sciences articles showed no growth from 1960 to 2001, which was similar to Iran's number of medical sci-

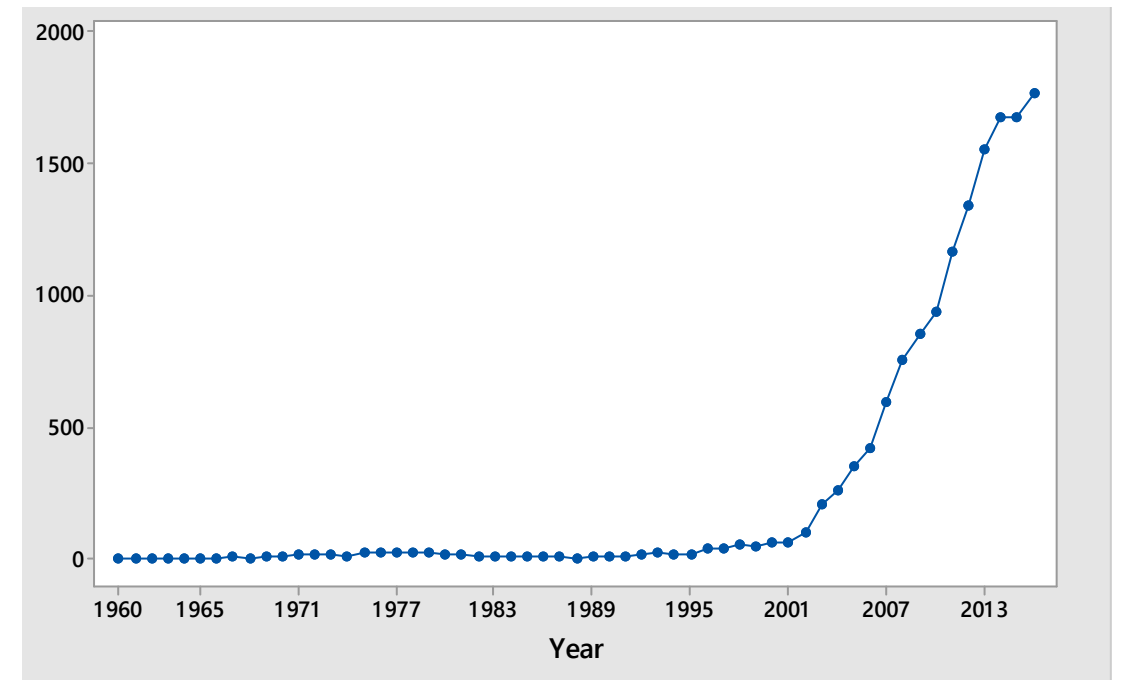

Diagram 2. Times series plot of Iran's number of medical sciences international collaboration articles from 1960 to 2016

Table 2. Iran's number of medical sciences articles and Iran's number of medical sciences international collaboration articles from 2017 to 2025

\begin{tabular}{|c|c|c|c|c|c|c|}
\hline \multirow[t]{3}{*}{ Year } & \multicolumn{3}{|c|}{ Iran's number of medical sciences articles } & \multicolumn{3}{|c|}{ Iran's number of medical sciences international collaboration articles } \\
\hline & \multirow[t]{2}{*}{ Forecast } & \multicolumn{2}{|c|}{ Confidence interval } & \multirow[t]{2}{*}{ Forecast } & \multicolumn{2}{|c|}{ Confidence interval } \\
\hline & & Lower & Upper & & Lower & Upper \\
\hline 2017 & 11919.4 & 11113.2 & 12753.9 & 1900.48 & 1721.51 & 2088.30 \\
\hline 2018 & 12237.4 & 10914.8 & 13635.6 & 2014.47 & 1771.26 & 2273.31 \\
\hline 2019 & 12559.5 & 10698.6 & 14569.5 & 2131.77 & 1817.19 & 2471.45 \\
\hline 2020 & 12885.8 & 10478.2 & 15542.2 & 2252.40 & 1861.50 & 2680.52 \\
\hline 2021 & 13216.3 & 10257.2 & 16550.0 & 2376.34 & 1905.12 & 2899.58 \\
\hline 2022 & 13551.0 & 10037.0 & 17591.4 & 2503.60 & 1948.51 & 3128.18 \\
\hline 2023 & 13889.9 & 9818.4 & 18665.8 & 2634.19 & 1991.90 & 3366.09 \\
\hline 2024 & 14232.9 & 9601.6 & 19772.9 & 2768.09 & 2035.43 & 3613.15 \\
\hline 2025 & 14580.2 & 9386.8 & 20912.4 & 2905.31 & 2079.20 & 3869.29 \\
\hline
\end{tabular}

Table 3. Accuracy measures and smoothing constants of models

\begin{tabular}{|c|c|c|c|c|c|c|c|c|c|}
\hline \multicolumn{5}{|c|}{ Iran's number of medical sciences articles model } & \multicolumn{5}{|c|}{ Iran's number of medical sciences international collaboration articles model } \\
\hline \multicolumn{2}{|c|}{ Accuracy measures } & \multicolumn{3}{|c|}{ Smoothing constants } & \multicolumn{2}{|c|}{ Accuracy measures } & \multicolumn{3}{|c|}{ Smoothing constants } \\
\hline$\alpha$ (level) & $\gamma($ trend) & MAPE & MAD & MSD & $\alpha($ level) & $\gamma($ trend) & MAPE & MAD & MSD \\
\hline 1.10271 & 0.47672 & 16.9807 & 1.5334 & 5.4784 & 1.10271 & 0.47672 & 16.9807 & 1.5334 & 5.4784 \\
\hline
\end{tabular}


ences international collaborative articles. This can be due to the fact that in this period, Iran was dealing with many events. Iran had a monarchy from 1960 to 1979 , and the literacy rate was very low (23). In 1979, the backwardness of the health of the country reached a point where Iran had the worst doctor-patient ratio, the highest infant and child mortality rates, and the lowest ratio of hospital beds to population in the Middle East (24). In 1979, the number of doctors in the country, both general practitioners and specialists of different disciplines was 14000 . A significant portion of this figure was foreign doctors (25). On the other hand, there was a breach of educational justice in geographical distribution and the literacy ratio between urban and rural populations was very different (26). The revolution of Iran occurred in February 1979. In April 1980, Iran's cultural revolution occurred, which led to the closure of universities and the deposal of hundreds of teachers and thousands of dissidents, leftists, and liberal students, and as a result, many intellectuals left Iran. Universities reopened after the cultural revolution in December 1982. In September 1980, the war with Iraq began and ended in August 1988. After the war, the long process of reconstruction was initiated (26-31).

However, Iran's medical sciences articles showed a trend of incremental growth from 2001 to 2016. The situation was the same for Iran's number of medical sciences international collaboration articles. This incremental growth can be attributed to the political, cultural, and scientific stability of the country from 2001 to 2016 . Nonetheless, this issue needs to be studied more. These results could also be due to the global incremental trend of national and international collaboration articles in the world from 2000 to 2016, and this reason confirms the incremental trend in India, Vietnam, Serbian, Morocco, Tunisia, Algeria, Australia, UK, and USA (18, 32-35). One study concerning national and international medical research of India showed that out of 50 medical institutes, 19 had average or above average annual growth rate in national research publications during 2003 and 2012. Only one institute had a negative growth rate for the same period (18). Results of that study indicated that the growth of medical research output has grown 2 folds during 2003 and 2012. In addition, 43 medical institutes have shown positive growth in their international collaboration during 2003 and 2012, and only 7 medical institutes had a negative growth rate for the same period. The research output of India with international collaboration has grown about 4 times during 2003 and 2012 (18). In our study, we did not investigate whether growth has occurred in all medical universities, and this point needs to be reviewed in another research.

Another study showed that the scientific publications of Vietnam increased during 1996 and 2013 (32). By the same token, articles published by Serbian researchers increased during 2006 and 2013. In this period, the annual number of published articles increased more than fivefold, the number of articles increased from 934 articles published in 2006 to 4855 in 2013 . One reason for this increase in Serbian articles was the change in the name of Serbia in 2006 (33). Two-thirds of all Serbian's articles were published in collaboration. Half of these collaborative articles were the results of collaboration between Serbian researchers and researchers from abroad. The distribution of those internationally collaborative articles indicated that the most collaborating countries with Serbian researchers were Germany and USA during 2006 and 2013 (33). In our study, we did not investigate which countries have been partners in different years with Iran, and there is a need for more research in this regard.

North African countries such as Morocco, Tunisia, and Algeria also showed an incremental trend in total publication articles from 1996 to 2012 (34). For the year 2000, SCImago indicated up to 1156 publications for Morocco, 738 for Tunisia, and 486 for Algeria. In 2012, the Moroccan researchers published 3282 articles, Algerians 3800, and Tunisians 5170 (34). In medical sciences, the output of the 3 countries remained very stable from 1996 to 2002 . Later, Tunisian outputs increased while the Moroccan output stagnated or even decreased until 2006, when it resumed increasing slowly (34). Another study showed that the rates of international collaboration in medical radiation science journals from Australia, the UK, and the USA increased during 2012 and 2014 (35).

This upward trend seems to continue into the future. In 2016, the percentage of Iran's international collaborative articles was 15.2, which will reach 19.9 in 2025. These results are consistent with the results of traditional Chinese medicine (36). One study analyzed the literature for traditional Chinese medicine articles in PubMed during 1995 to 2014 and predicted the number of the articles in future. Findings revealed that in 1995, 711 articles were published, while in 2013, the number of publications increased to 3162 . Based on the rising trend, it can be predicted that the number of articles on traditional Chinese medicine will continue growing at a high rate in the future (36). Many nations participate in international collaboration (37). Thus, it can be stated that international collaboration grows in science, but little agreement exists about dynamics of growth at the discipline level. Some suggest that disciplines differ in their collaborative tendencies (38).

Our findings indicated that the number of articles has been increased in Iran with international cooperation. However, an increase or decrease in Iran's articles without international cooperation has to be investigated.

The number of articles and international collaborative articles in medical sciences was extracted from SCOPUS database. However, such data may not be the same in different databases. Therefore, it is suggested that data be extracted from the Web of Science.

\section{Conclusion}

An investigation into the trends of Iran's number of medical sciences international collaboration articles showed incremental growth. The number of articles has been increased in Iran with international cooperation. However, the increase or decrease of Iran's articles without international cooperation needs to be investigated. 


\section{Acknowledgements}

This article was extracted from a research project (number: 395564). We thank the Deputy of Education of the Ministry of Health and Medical Education and Vice Chancellor for Research of Isfahan University of Medical Sciences for supporting this project.

\section{Conflict of Interests}

The authors declare that they have no competing interests.

\section{References}

1. Groff L, Smoker P. Introduction to Future Studies. 2003. Available from: http://www.csudh.edu/global_options/IntroFS.HTML

2. Bell W. An overview of futures studies. In: Slaughter R (Ed). The knowledge base of futures studies: Foundations. Hawthorn, Victoria: DDM Media; 1996. [Cited 2016 February 24] Available from: http://www.wendellbell.com/wp-content/uploads/2015/10/An-over view- of-FS_1996.pdf

3. Dator J. What futures studies is, and is not. [Cited 2016 February 24] Available from: http://www.futures.hawaii.edu/publications/futuresstudies/WhatFSis1995.pdf

4. Shrivats V, Bhattacharya S. Forecasting the trend of international scientific collaboration. Scientometrics. 2014; 101: 1941-1954.

5. Groff L, Smoker P. Introduction to future studies. [Cited 2016 February 24] Available from:

http://www.itari.in/categories/futurestudies/IntroductiontoFutureStudie s.pdf

6. Hallonsten O. Continuity and Change in the Politics of European Scientific Collaboration. JCER. 2012; 8(3): 299-318.

7. Leydesdorff L, Wagner C, Park HW, Adams J. International Collaboration in Science: The Global Map and the Network. El Prof de la Info (2013, in press)

8. Narin F, Whitlow ES. Measurement of scientific cooperation and coauthorship in EEC-related area of science. Commission of the European community; 1990.

9. Franklin MN. The community of science in Europe: Preconditions for Research Effectiveness in European Community Countries. Gower, Brussels; 1998.

10. Kwon KS, Park HW, So M, Leydesdorff L. Has Globalization Strengthened South Korea's National Research System? National and International Dynamics of the 18 Triple Helix of Scientific Coauthorship Relationships in South Korea. Scientometrics. 2012; 90(1):163-175.

11. Zhou P, Glänzel W. In-depth analysis on China's international cooperation in science. Scientometrics. 2010;82(3):597-612.

12. SCIMago journal and country ranking. Country search. United States. [Cited 2016 February 24] Available from: http://www.scimagojr.com/countrysearch.php?country=US\&area=0

13. SCIMago journal and country ranking. Country search. China. [Cited 2016 February 24] Available from: http://www.scimagojr.com/countrysearch.php?country $=\mathrm{CN} \&$ area $=0$

14. SCIMago journal and country ranking. Country search. United Kingdom. [Cited 2016 February 24] Available from: http://www.scimagojr.com/countrysearch.php? country $=$ GB\&area $=0$

15. SCIMago journal and country ranking. Country search. Germany. [Cited 2016 February 24] Available from: http://www.scimagojr.com/countrysearch.php?country=DE\&area=0

16. SCIMago journal and country ranking. Country search. Japan. [Cited 2016 February 24] Available from: http://www.scimagojr.com/countrysearch.php? country=JP\&area $=0$

17. SCIMago journal and country ranking. Country search. Iran. [Cited 2016 February 24] Available from: http://www.scimagojr.com/countrysearch.php? country $=$ IR\&area $=0$

18. Kaur H, Mahajan P. National versus international growth of medical research

of India: a case study. Scientometrics. 2015; 105: 973-989.

19. Bowerman BL, O' Connell RT. Forecasting and Time Series: An Applied Approach. 3rd ed. Duxbury Press; 1993.

20. Beaver D, Rosen R. Studies in scientific collaboration: Part I--The professional origins of scientific co-authorship. Scientometrics. 1978; 1: 65-84.
21. Georghiou L. Global cooperation in research. Research Policy. 1998; 27: 611-626.

22. Katz JS, Martin BR. What is research collaboration? Research Policy. 1997; 26: 1-18.

23. Mohamadi M. [A review of Iran's foreign policy or decision making in the Pahlavi era dominated system]. Tehran: Dadgostar; 1998. [Persian]

24. Tadayon A (Translator). [Fragile Resistance: A History of Social Transformation in Iran]. Furan J (Author). Tehran: rasa; 2006. [Persian]

25. Moradian M. [Thirty-year workbook of the Islamic Republic of Iran]. Soureh. 2009;(42):27. [Persian]

26. Rafiei H. [Economic infrastructure before and after the Islamic Revolution in Iran]. Tehran: Islamic Revolution Documents Center; 2010. [Persian]

27. Keddie NR, Richard Y. Modern Iran: roots and results of revolution. New haven \& London: Yale University Press; 2006.

28. Sobhe K. Education in Revolution: Is Iran Duplicating the Chinese Cultural Revolution? Comparative Education. 1982; 18(3): 271-280.

29. Momen M. An Intoroduction to Shi'i Islam, the History and Doctrines of Twelver Shi'ism. New haven \& London: Yale University Press; 1985.

30. Gheissari A, Vali N. Democracy in Iran: History and the Quest for Liberty. $1^{\text {st }}$ ed. Oxford University Press; 2009.

31. Shahriari H. [Discourse Analysis of Iran and Iraq War]. Res Theo Polit. 2015;(15):193-222. [Persian]

32. Manh HD. Scientific publications in Vietnam as seen from Scopus during 1996-2013. Scientometrics. 2015;105:83-95.

33. Ivanovic D, Fu H, Ho Y. Publications from Serbia in the Science Citation Index Expanded: a bibliometric analysis. Scientometrics. 2015;105:145-160.

34. Medina F. The output of researchers in Morocco compared to some North African countries from 1996 to 2012, and its relationship to governmental major decisions on higher education and scientific research. Scientometrics. 2015; 105: 367-384.

35. Denham G, Allen C, Platt J. International collaboration in medical radiation science. J Med Radiat Sci. 2016;63(2):75-80.

36. Huang Y, Zhou M, Deng Q, Zhang J, Zhou P, Shang X. Bibliometric analysis for the literature of traditional Chinese medicine in PubMed. Scientometrics. 2015;105:557-566.

37. Bornmann L, Wagner C, Leydesdorff L. BRICS countries and scientific excellence: A bibliometric analysis of most frequently cited papers. JASIST. 2015; 66(7): 1507-1513.

38. Wagner CS, Whetsell TA, Leydesdorff L. Growth of International Collaboration in Science: Revisiting Six Specialties. Scientometrics; 2017; 110(3): 1633-1652. 